\title{
Short Communication on "MATS: Global Coverage Estimates for 4CMenB, a Novel Multicomponent Meningococcal B Vaccine"
}

\author{
Medini $\mathbf{D}^{1^{*}}$ and Vernikos $\mathbf{G}^{2}$ \\ ${ }^{1}$ GlaxoSmithKline, Vaccines Research \& Development Department, Via Fiorentina 1, Italy \\ ${ }^{2}$ GlaxoSmithKline, Medical Affairs Department, Kifisias Avenue 266, Athens, Chalandri, Greece
}

*Corresponding author: Duccio Medini, GlaxoSmithKline, Vaccines Research \& Development Department, Via Fiorentina 1, 53100 Siena, Italy, Tel: +30210688 2216; E-mail: georgios.x.vernikos@gsk.com.

Received date: Oct 12, 2015; Accepted date: Oct 30, 2015; Published date: Oct 02, 2015

Copyright: ( 2015 Medini D, et al. This is an open-access article distributed under the terms of the Creative Commons Attribution License, which permits unrestricted use, distribution, and reproduction in any medium, provided the original author and source are credited.

\begin{abstract}
Meningococcal antigen typing system (MATS) - the golden standard in many reference labs worldwide-for rapid, reproducible and accurate (conservative nonetheless) prediction of Bexsero strain coverage, can pave the way towards a data-driven decision making of national immunization technical advisory groups (NITAGs), allowing indirect comparison of immunization policies across countries and regions leading to rapid adaptation of public health policy decisions based on worldwide datasets. The recent milestone of the UK national infant routine recommendation and reimbursement, paves the way for other nations to follow, bringing closer the pediatric community to the vision of a meningitis free world.
\end{abstract}

\section{Description}

Since September the $1^{\text {st }}$, infants in the UK are routinely vaccinated with Bexsero, marking the start of the world's first nationwide, publicly funded menB vaccination program. Experts and charities have wholeheartedly welcomed the decision to include Bexsero in routine childhood vaccination schedule.

Under this program, which was agreed between GSK and the UK's Department of Health in March 2015, infants are vaccinated at 2 and 4 months of age, with a booster dose administered at 12-13 months; catch-up for infants of 3 and 4 months of age is also offered free of charge.

This agreement follows recommendations issued in March 2014 by the joint committee on vaccination and immunization (JCVI) calling for Bexsero to be introduced into the infant immunization program, starting at the second month of age.

Towards the same direction of Bexsero public funding, regional reimbursement is currently implemented in Italy (more than 9 regions) while sub-regional funding is applicable in Canada in the region of Saguenay Lac St. Jean of Quebec.

In many countries quadrivalent and or monovalent meningococcal vaccinations are reimbursed, although serogroup A, C, W135, Y epidemiology is significantly lower than that of men B. Indeed, recently Yamashiro et al. [1], discussed this point of view in the case of Ontario (CA), pointing out that the rationale of Bexsero's exclusion from reimbursement scheme is difficult to explain and has perhaps potential legal implications. This is because routine recommendations and funding are currently in place for meningococcal serogroups that cause significantly less disease in Canada, which in return inflates parents' confusion about which vaccination (A, C, W135, Y or B) is of higher priority based on the current epidemiological landscape of their region.

Since January 2013, that EMEA approved Bexsero in Europe, the vaccine has received more than 20 clinical recommendations in the UK (JCVI), Czech Republic (NIKO), Australia (ATAGI), Greece (Greek Pediatric Society), Austria (BMG), Italy (Board of calendario per la Vita), Ireland (NIAC), Spain (AEMPS), Portugal (SIP and SPP), Germany (DTG, SIKO, DAKJ), Canada (NACI), Hungary (NEC), and Poland (GIS-MOH) for active immunization of individuals from 2 months of age and older against invasive meningococcal disease (IMD) caused by Neisseria meningitides serogroup B.

In the US, since January 2015 Bexsero is registered in adolescents and young adults from 10 years through 25 years of age, making Bexsero the first men B vaccine with dual approval from EMEA and FDA. Worldwide more than 1.500 .000 doses have been distributed and 37 countries have approved Bexsero so far; 10.000 pediatricians have already implemented Bexsero vaccinations.

Medini et al. [2], published the most recent update of the meningococcal antigen typing system (MATS) - the golden standard in many reference labs worldwide-for rapid, reproducible and accurate (conservative nonetheless) prediction of Bexsero strain coverage. The authors briefly touched a very pivotal viewpoint that MATS can pave the way towards a data-driven decision making of national immunization technical advisory groups (NITAGs), allowing indirect comparison of immunization policies across countries and regions leading to rapid adaptation of public health policy decisions based on worldwide datasets.

Indeed post-implementation surveillance strives and calls-for highly standardized platforms that make feasible to share and cross-compare heterogeneous epidemiological landscapes of different countries with the aim to pursue data-driven decision making for nationwide recommendation and reimbursement programs by NITAGs. MATS platform is ideal for this type of inter-country standardization providing on-going snapshot of Bexsero impact on meningococcal populations and more importantly predict real-time changes in vaccine efficacy even before they actually occur.

The recent milestone of the UK national infant routine recommendation and reimbursement, paves the way for other nations to follow, bringing closer the pediatric community to the vision of ameningitis-free world.

Of interest is the fact that the UK led the nationwide, publicly funded MenB vaccination although in terms of MATS predicted percentage coverage it does not sit in the highest end of the spectrum. This means that other countries should expect a risk/benefit ratio even 
Citation: Medini D, Vernikos G (2015) Short Communication on "MATS: Global Coverage Estimates for 4CMenB, a Novel Multicomponent Meningococcal B Vaccine". J Vaccines Vaccin 6: 296. doi:2157-7560.1000296

Page 2 of 2

more favorable than UK took in deciding for nationwide vaccination campaigns.

As soon as other countries implement nationwide funded Bexsero vaccination programs, MATS and strain genotyping will be powerful tools for "comparative surveillance", to adjust and fine-tune vaccination strategies in terms of catch-up need, immunization of adolescent cohort(s), number of required doses in infants, booster dose need, optimal dose intervals, etc.) leveraging on cross-country real-time outcomes.

The next big challenge is to set the basis for a standardized, uniform, consistent and data-driven decision making that is scientifically and ethically waterproof, living no space for political and/or financial distortion of new antigen entry in existing national immunization programs (NIPs).
Besides, as WHO puts this, excluding clean water, nothing but vaccination has so much impact in reducing mortality and increasing life expectancy of the human kind. Vaccination is a human right, and deserves humanitarian, rather than technocratic perspective of decision making.

\section{References}

1. Yamashiro H, Cutcliffe N, Dobson S, Fisman D, Gold R (2015) The role of pediatricians as key stakeholders in influencing immunization policy decisions for the introduction of meningitis B vaccine in Canada: The Ontario perspective. Can J Infect Dis Med Microbiol 26: 183-190.

2. Medini D, Stella M, Wassil J (2015) MATS: Global coverage estimates for $4 \mathrm{CMenB}$, a novel multicomponent meningococcal B vaccine. Vaccine 33 : 2629-2636. 